\title{
Effect of Steroid-Soaked Gelatin Sponge on Soft Tissue Swelling Following Anterior Cervical Discectomy and Fusion: A Radiographic Analysis
}

\author{
Joshua Schroeder ${ }^{*}$, Joseph Weinstein ${ }^{*}$, Stephan Nikolaus Salzmann, Janina Kueper, \\ Jennifer Shue, Andrew Anthony Sama, Federico Pablo Girardi \\ Spine and Scoliosis Service, Hospital for Special Surgery, New York, NY, USA
}

\begin{abstract}
Study Design: Retrospective radiological review and analysis of 79 patients who underwent primary anterior cervical discectomy and fusion (ACDF) of 2 or 3 levels between 2011 and 2013.

Purpose: This study aimed to determine the effect of the local placement of a steroid-soaked gelatin sponge after ACDF on prevertebral soft tissue swelling.

Overview of Literature: Although ACDF has become a popular choice for cervical fusion, the surgical involvement of the delicate anatomy of the neck frequently results in tissue irritation and edema. Swelling of the prevertebral soft tissue may consequently lead to mild-to-severe complications, ranging from dysphonia to dyspnea.

Methods: Out of the 79 patients who underwent primary ACDF, 52 received a gelatin sponge soaked with $40 \mathrm{mg}$ of Depo-Medrol placed adjacent to the operated cervical levels. Prevertebral soft tissue swelling was detected using postoperative lateral X-ray. The radiographic values were compared to those of 27 patients who did not receive the treatment.

Results: Soft tissue swelling was markedly decreased in patients who received the placement of the steroid-soaked gelatin sponge next to their fused levels after surgery compared with that in patients who did not receive it. No complications were documented with the use of steroids.

Conclusions: The placement of a steroid-soaked gelatin sponge markedly reduces postoperative soft tissue swelling following 2- or 3-level primary ACDF.
\end{abstract}

Keywords: Cervical vertebrae; Deglutition disorders; Dysphonia; Absorbable gelatin sponge; Methylprednisolone

\section{Introduction}

Over the past decades, anterior cervical discectomy and fusion (ACDF) has become one of the most common surgical procedures to decompress and fuse the cervical spine. The number of ACDF procedures performed has dramati- cally increased, particularly in the patients aged $\geq 65$ years [1]. The anterior approach to the cervical spine challenges the surgeon to navigate through the complex anatomy of the anterior aspect of the neck, bearing the hazards of injury to the neural and vascular structures. Nonetheless, ACDF has been shown to result in a lower rate of perioper-

Received Sep 29, 2017; Revised Dec 11, 2017; Accepted Dec 17, 2017

Corresponding author: Federico Pablo Girardi

Spine and Scoliosis Service, Hospital for Special Surgery, 535 East 70th St., New York, NY 10021, USA

Tel: +1-212-606-1559, Fax: +1-212-774-2870, E-mail: girardif@hss.edu

*These two authors contributed equally to this work. 
ative complications and mortality and a shorter duration of hospital stay than posterior cervical discectomy and fusion [2]. This finding has significantly promoted the popularity of ACDF in the surgical community.

Due to the disruption and distension of the delicate anterior cervical structures surrounding the anterior aspect of the cervical spine, tissue inflammation may result in complications that can seriously affect the patient. Pharyngeal edema resulting from the tissue inflammatory reaction may constrict the esophagus and trachea, culminating in dysphagia or dyspnea [3-6]. The severity of dyspnea corresponds with the degree of compression of the trachea due to swelling of the surrounding tissues. In severe cases, this swelling may lead to emergent intubations to secure the airway [4,7]. Contrarily to dyspnea, no direct correlation of the degree of soft tissue swelling with the severity of dysphagia has been reported $[4,8]$.

Few treatment modalities for soft tissue swelling have been studied. The most prominent option is the use of steroids to inhibit the naturally occurring inflammatory cascade, thereby reducing both postoperative pain and edema [9]. The limited use of steroids through epidural injections, intravenous administration, or local placement on a carrier postoperatively in both the lumbar and cervical spine has been analyzed over the past few years [10-15]. Several studies have reported a decrease in the opioid usage, duration of hospital stay, and incidence of neurological adverse sequelae as well as a decrease in soft tissue swelling in the cervical spine following steroid administration $[11,13,15]$. Some other studies have reported no measurable influence of the steroids on functional recovery, pain, or the length of intubation of the patient $[10,12,14]$.

Encouraged by the lack of complications reported in association with steroid application after cervical surgery, this study aimed to determine whether the direct placement of a gelatin sponge soaked in low-dose steroids decreases the radiological degree of soft tissue swelling $[14,15]$.

\section{Materials and Methods}

\section{Ethical statement}

For the patients presented in this study, a waiver of patient informed consent and U.S. Health Insurance Portability and Accountability Act authorization was sought. Infor- mation contained in this study contains no personal identifiers to ensure patient confidentiality and protections. Under these provisions, the Institutional Review Board at Hospital for Special Surgery provided approval of this study (IRB approval no., \#2014-356).

\section{Patients}

Between January 2011 and January 2013, 52 patients underwent 2- or 3-level primary ACDF with local steroid placement. The indication for surgery was degenerative cervical disease with radiculopathy and/or myelopathy. These patients were matched with 27 patients who underwent 2- or 3-level primary ACDF but were not treated with local steroids. The fusion area of the included cases ranged from C3 to T1. To shortlist patients with significant symptoms and prevertebral soft tissue swelling, only those patients who underwent 2 or 3 levels of fusion were included in this study. There were no differences in the clinical indications for the use of steroid-soaked Gelfoam sponge (Pfizer, New York, NY, USA) versus no steroid use. The local application of steroid was based on the preference of the surgeon. We compared the differences between the results obtained by surgeons who used steroids in all anterior cervical fusion cases and those who did not. The charts and images of the patients were reviewed. Data on demographic parameters, comorbidities, operative details, and postoperative course were collected. Dysphagia, clinically defined as difficulty in swallowing that is expressed as a novel complaint by patients postoperatively, was queried throughout the patients' stay at the hospital as well as during their postoperative visits. All patients underwent surgery by two senior spine surgeons, with a joint experience of $>25$ years in spine surgery.

\section{Intervention}

The exposure was performed with the standard SmithRobinson approach to the anterior spine [16]. Decompression of the levels was performed, and cages were placed in the disc spaces. The spine was stabilized by locked plates (Gradient Plus Anterior Cervical Plate systems; NuVasive, San Diego, CA, USA). After irrigation of the wound, a piece of Gelfoam sponge (Pfizer), cut to span the full length of the operated levels in each patient, was soaked with a steroid solution containing $40 \mathrm{mg}$ Depo-Medrol and placed adjacent to the operated levels before closure. 
The wound was closed over a Penrose drain that was removed on postoperative day 1 or 2 in all patients.

\section{Assessment of swelling}

The soft tissue swelling was evaluated using standing lateral cervical X-rays on postoperative day 2 (range, 1-5 days). The measurements were taken over the location of the largest amount of swelling at each cervical level operated upon. Two fellowship-trained spinal surgeons independently completed the measurements to confirm the interobserver reliability of the results.

\section{Statistical Analysis}

Analyses were performed using PASW SPSS statistical software ver. 18.0 (SPSS Inc., Chicago, IL, USA). The Student $t$-test and chi-square test were used to compare the cohort. Scores were presented as mean \pm standard deviation. Statistical significance was set at $p<0.05$.

\section{Results}

\section{Patients}

The average age of the treatment group was 51.0 years (range, 27-72 years) versus an average age of 55.6 years (range, 39-82 years) of the control group. Gender distribution did not differ between the groups (Table 1). The mean operative time was 224.7 minutes (range, 165-285 minutes) for the sponge treatment group and 274.7 minutes (range, 210-312 minutes) for the control group $(p<0.05)$. No preoperative dysphagia or dysphonia was documented in either group.

\section{Soft tissue swelling}

Postoperative prevertebral soft tissue swelling was found to be significantly reduced in patients who received the Depo-Medrol-soaked Gelfoam sponge (Pfizer). The mean soft tissue swelling was found to be lower in the sponge treatment group than that in the control group $(11.21 \mathrm{~mm}$ versus $15.47 \mathrm{~mm}$ at $\mathrm{C} 4[p<0.05], 13.54 \mathrm{~mm}$ versus 17.96 $\mathrm{mm}$ at $\mathrm{C} 5[p<0.05], 15.45 \mathrm{~mm}$ versus $18.21 \mathrm{~mm}$ at C6 [ $p<0.05]$, and $15.72 \mathrm{~mm}$ versus $17.92 \mathrm{~mm}$ at $\mathrm{C} 7[p<0.05])$. The C3 level demonstrated increased soft tissue swelling in the sponge treatment group at $10.28 \mathrm{~mm}$ versus 10.18 $\mathrm{mm}$, which was not statistically significant (Table 2). Fig. 1 shows the soft tissue swelling measurements of two representative cases.

\section{Clinical outcomes}

Three patients in the control group experienced postoperative dysphagia compared with none in the steroid treatment group $(p<0.01)$. Two of these patients required additional attention and received nutritional supplementation.

Table 1. Patient demographics

\begin{tabular}{lccc} 
Variable & Steroid group & Control group & Significance \\
Mean age (yr) & 51.0 & 55.6 & Not significant \\
Gender (male/female) & $29 / 23$ & $14 / 13$ & Not significant \\
Body mass index $\left(\mathrm{kg} / \mathrm{m}^{2}\right)$ & 26.71 & 26.96 & Not significant \\
\hline
\end{tabular}

Table 2. Soft tissue swelling in the steroid and control groups

\begin{tabular}{lccc} 
Level & Steroid group & Control group & Significance \\
C3 $(\mathrm{mm})$ & $10.28 \pm 3.74$ & $10.18 \pm 3.53$ & Not significant \\
C4 $(\mathrm{mm})$ & $11.21 \pm 3.83$ & $15.47 \pm 6.01$ & $p<0.05$ \\
C5 $(\mathrm{mm})$ & $13.54 \pm 3.98$ & $17.96 \pm 4.86$ & $p<0.05$ \\
C6 $(\mathrm{mm})$ & $15.45 \pm 3.45$ & $18.21 \pm 3.61$ & $p<0.05$ \\
C7 $(\mathrm{mm})$ & $15.72 \pm 3.34$ & $17.92 \pm 3.06$ & $p<0.05$ \\
\hline
\end{tabular}

Values are presented as mean \pm standard deviation. 

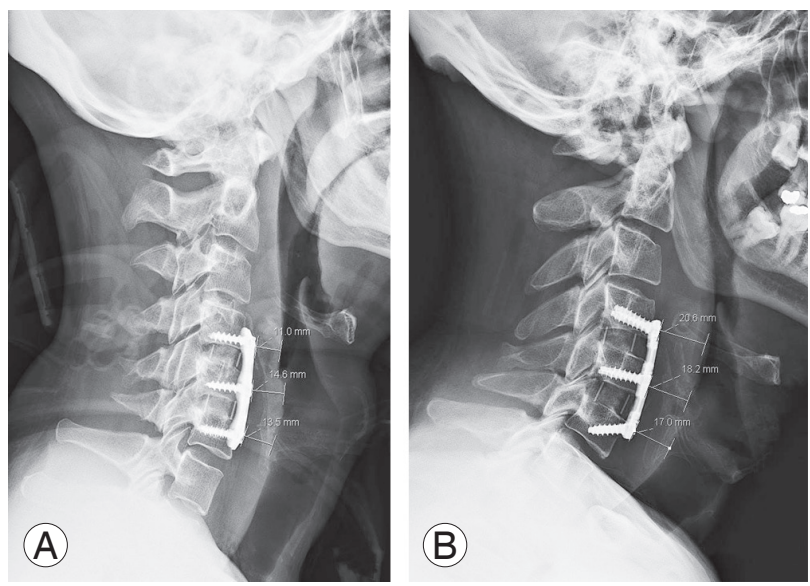

Fig. 1. Standing lateral X-ray image on the postoperative day 2 after anterior cervical discectomy and fusion. The soft tissue swelling in the patient receiving local steroids $(\mathbf{A})$ is significantly lesser than that in the control patient (B).

No dyspnea was noted in either group. No complications associated with the local administration of the low-dose steroids occurred in the treatment group. At 30-month follow-up, no cases of revision surgery for pseudarthrosis were identified in either group.

\section{Discussion}

The incidence of major systemic complications after ACDF has been reported to be as low as 3.3\% [17]. In contrast, the incidence of minor complications related to the anterior surgical approach of the procedure has been reported to be relatively high. The most common minor complication was found to be dysphagia, with a reported incidence of $5 \%-71 \%[18,19]$. The risk of dysphagia has been correlated with the amount of pressure placed on the soft tissues of the neck during the procedure, leading to esophageal ischemia and reperfusion injuries [20]. In this study, postoperative prevertebral soft tissue edema and swelling were significantly reduced in patients receiving a local application of Depo-Medrol-soaked Gelfoam sponge following their 2- to 3-level ACDF compared with those in the control cases. In addition, no cases of dysphagia were documented in the steroid treatment group compared with the three cases of dysphagia that needed medical intervention in the control group.

Previous studies have examined both the systemic as well as local administration of steroids for combating the clinical sequelae of prevertebral soft tissue swelling.
Conclusions regarding the efficacy of the treatment modality are contradictory. Several prospective studies have examined the effect of intravenous administration of steroids on the incidence of ear, nose, and throat (ENT) complications such as lesions, swelling, or compression of the pharyngeal wall, arytenoids, vocal cords, and trachea. Song et al. [21] and Pedram et al. [22] administered intravenous methylprednisolone following ACDF and reported favorable outcomes with respect to the extent of prevertebral soft tissue swelling and the lesions identified through postoperative nasofibroscopic examination, respectively. In contrast, Emery et al. [14] reported no decrease in the incidence of edema and delayed extubation in patients who received intravenous dexamethasone and were undergoing 2- or 3-level anterior cervical corpectomy procedures with anterior strut graft reconstruction. Lee et al. [15] were the first to analyze the effect of locally administered steroids in the form of triamcinolone placed in the retropharyngeal space on a morcellized collagen sponge. In their group of 1- and 2-level ACDF patients, prevertebral soft tissue swelling and odynophagia were found to be significantly reduced in patients receiving steroid-soaked sponge. The incidence of complications as well as the readmission and revision rates have been reported to be significantly higher in patients undergoing a multi-level versus a single-level ACDF; hence, we decided to examine patients undergoing 2- to 3-level ACDFs [23].

The main limitation of this study, in addition to the inherent limitations of a retrospective study, was that the surgery time in the control group was longer than that in the intervention group, which increased the likelihood of a confounder effect on the prevertebral soft tissue swelling. However, because the cases were all primary and 2-3-level cases, the $18 \%$ increase in the time could not explain the large differences in soft tissue swelling. Possible explanations for the observed differences in the operation time could be due to patient factors such as comorbidities or anatomical abnormalities as well as differences in the surgical training of the surgeon. However, because both the surgeons were seniors and fellowship-trained with similar surgical volumes, the surgical technique for the procedure did not significantly differ between the two surgeons. Another limitation of this study was that the postoperative standing lateral cervical X-rays were not performed on the same day as that of the operation. Institutional perioperative imaging acquisition follows a patient-care protocol, where standing radiographs are 
obtained when the patients can stand and tolerate the imaging examination. In this study, the radiographic swelling was measured on films taken at a mean of 2 days after the surgery (range, 1-5 days). Therefore, the amount of swelling could have varied based on the timing of the radiograph. In addition, dysphagia, although clinically documented by an ENT specialist, was not radiographically confirmed using barium-swallow esophagography.

\section{Conclusions}

The use of local steroids, placed on a collagen sponge, decreases the soft tissue swelling after 2- or 3-level primary ACDF. This finding may contribute to the prevention of dysphagia development after surgery. In the future, prospective studies of the effect of steroid administration on the amount of postoperative dysphagia development are warranted.

\section{Conflict of Interest}

No potential conflict of interest relevant to this article was reported.

\section{Author Contributions}

The individual authors contributed in the following manner: Schroeder J: study design, data acquisition, data interpretation, manuscript composition; Weinstein J: study design, data acquisition, data interpretation, manuscript composition; Salzmann SN: data interpretation, manuscript editing; Kueper J: data interpretation, manuscript editing; Shue J: data interpretation, manuscript editing; Sama AA: study design, manuscript editing; and Girardi FP: study design, manuscript editing, supervision.

\section{References}

1. Marawar S, Girardi FP, Sama AA, et al. National trends in anterior cervical fusion procedures. Spine (Phila Pa 1976) 2010;35:1454-9.

2. Memtsoudis SG, Hughes A, Ma Y, Chiu YL, Sama AA, Girardi FP. Increased in-hospital complications after primary posterior versus primary anterior cervical fusion. Clin Orthop Relat Res 2011;469:649-57.

3. Martin RE, Neary MA, Diamant NE. Dysphagia following anterior cervical spine surgery. Dysphagia
1997;12:2-8.

4. Sagi HC, Beutler W, Carroll E, Connolly PJ. Airway complications associated with surgery on the anterior cervical spine. Spine (Phila Pa 1976) 2002;27:949-53.

5. Smith-Hammond CA, New KC, Pietrobon R, Curtis DJ, Scharver CH, Turner DA. Prospective analysis of incidence and risk factors of dysphagia in spine surgery patients: comparison of anterior cervical, posterior cervical, and lumbar procedures. Spine (Phila $\mathrm{Pa}$ 1976) 2004;29:1441-6.

6. Riley LH 3rd, Vaccaro AR, Dettori JR, Hashimoto R. Postoperative dysphagia in anterior cervical spine surgery. Spine (Phila Pa 1976) 2010;35(9 Suppl):S76-85.

7. Schroeder J, Salzmann SN, Hughes AP, Beckman JD, Shue J, Girardi FP. Emergent reintubation following elective cervical surgery: a case series. World J Orthop 2017;8:465-70.

8. Kepler CK, Rihn JA, Bennett JD, et al. Dysphagia and soft-tissue swelling after anterior cervical surgery: a radiographic analysis. Spine J 2012;12:639-44.

9. Floman Y, Zor U. Mechanism of steroid action in inflammation: inhibition of prostaglandin synthesis and release. Prostaglandins 1976;12:403-13.

10. Lavyne MH, Bilsky MH. Epidural steroids, postoperative morbidity, and recovery in patients undergoing microsurgical lumbar discectomy. J Neurosurg 1992;77:90-5.

11. Mirzai H, Tekin I, Alincak H. Perioperative use of corticosteroid and bupivacaine combination in lumbar disc surgery: a randomized controlled trial. Spine (Phila Pa 1976) 2002;27:343-6.

12. Debi R, Halperin N, Mirovsky Y. Local application of steroids following lumbar discectomy. J Spinal Disord Tech 2002;15:273-6.

13. Rasmussen S, Krum-Moller DS, Lauridsen LR, et al. Epidural steroid following discectomy for herniated lumbar disc reduces neurological impairment and enhances recovery: a randomized study with twoyear follow-up. Spine (Phila Pa 1976) 2008;33:202833.

14. Emery SE, Akhavan S, Miller P, et al. Steroids and risk factors for airway compromise in multilevel cervical corpectomy patients: a prospective, randomized, double-blind study. Spine (Phila Pa 1976) 2009;34:229-32.

15. Lee SH, Kim KT, Suk KS, Park KJ, Oh KI. Effect of 
retropharyngeal steroid on prevertebral soft tissue swelling following anterior cervical discectomy and fusion: a prospective, randomized study. Spine (Phila Pa 1976) 2011;36:2286-92.

16. Jain KK. Anterior approach to the cervical spine. Can Med Assoc J 1974;111:49-50.

17. Gruskay JA, Fu M, Basques BA, et al. Factors affecting length of stay and complications after elective anterior cervical discectomy and fusion: a study of 2164 patients from the American College of Surgeons National Surgical Quality Improvement Project Database (ACS NSQIP). Clin Spine Surg 2016;29:E3442.

18. Fountas KN, Kapsalaki EZ, Nikolakakos LG, et al. Anterior cervical discectomy and fusion associated complications. Spine (Phila Pa 1976) 2007;32:2310-7.

19. Rihn JA, Kane J, Albert TJ, Vaccaro AR, Hilibrand AS. What is the incidence and severity of dysphagia after anterior cervical surgery? Clin Orthop Relat Res 2011;469:658-65.
20. Heese O, Fritzsche E, Heiland M, Westphal M, Papavero L. Intraoperative measurement of pharynx/ esophagus retraction during anterior cervical surgery: part II: perfusion. Eur Spine J 2006;15:1839-43.

21. Song KJ, Lee SK, Ko JH, Yoo MJ, Kim DY, Lee KB. The clinical efficacy of short-term steroid treatment in multilevel anterior cervical arthrodesis. Spine J 2014;14:2954-8.

22. Pedram M, Castagnera L, Carat X, Macouillard G, Vital JM. Pharyngolaryngeal lesions in patients undergoing cervical spine surgery through the anterior approach: contribution of methylprednisolone. Eur Spine J 2003;12:84-90.

23. Veeravagu A, Cole T, Jiang B, Ratliff JK. Revision rates and complication incidence in single- and multilevel anterior cervical discectomy and fusion procedures: an administrative database study. Spine J 2014;14:1125-31. 